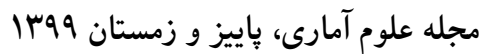

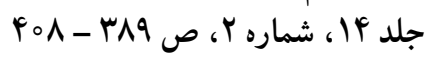

DOI: $10.29252 /$ jss.14.2.389

\section{معيار نادرستى بر مبناى تابع مفصل بقاء}

$$
\begin{aligned}
& \text { سيده تكتم حسينى و جعفر احمدى } \\
& \text { كروه آمار، دانشكده علوم رياضى، دانشگاه فردوسى مشهد }
\end{aligned}
$$

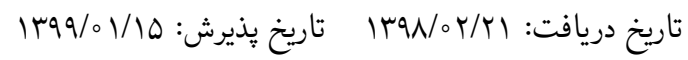

جكيده: در اين مقاله، با استفاده از ايده اندازه نادرستى در نظريه اطلاع، معيارهاى نادرستى باقيمانده

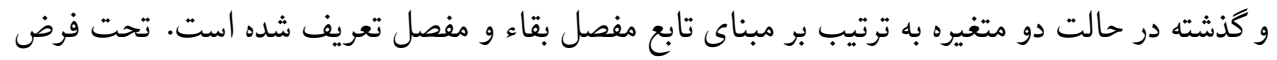

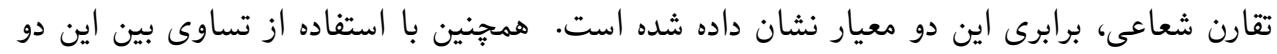

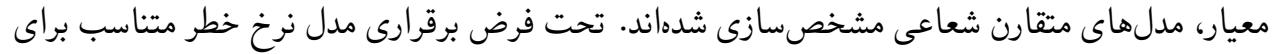

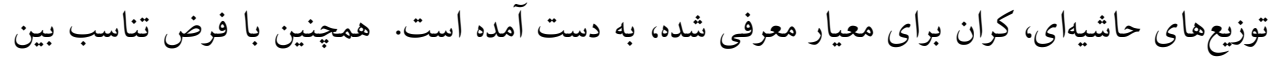

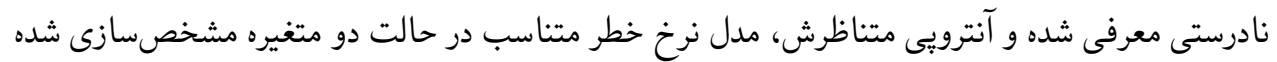

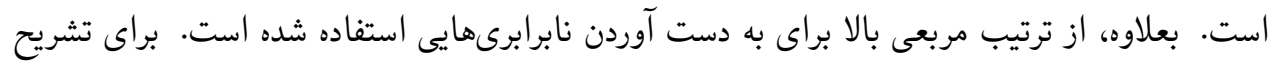

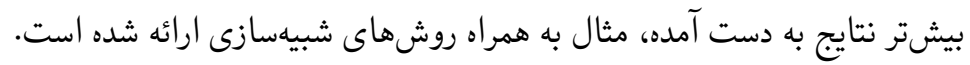

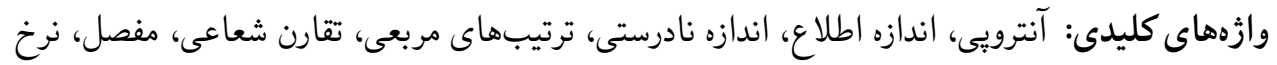
خطر (معكوس) متناسب.

\section{|}

اندازه نادرستى ' يكى از معيارهاى مهم در نظريه اطلاع به شمار مىآيد كه توسط كريج (1991) براى دو

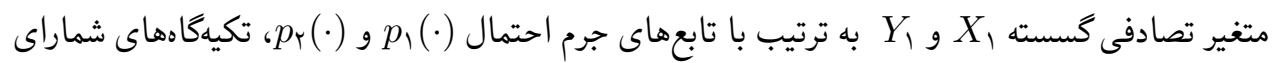
آدرس الكترونيكى نويسنده مسئول مقاله: سيده تكتم حسينى،

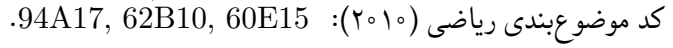

\footnotetext{
${ }^{1}$ Inaccuracy measure
} 
معيار نادبرستى بر مبناى تابع مفصل بقاء

$r q 0$

$I\left(X_{1}, Y_{\uparrow}\right)=-\sum_{x \in S_{X_{1}} \cap S_{Y_{1}}} p_{\uparrow}(x) \log p_{\Upsilon}(x)$ تعريف شده است. نات

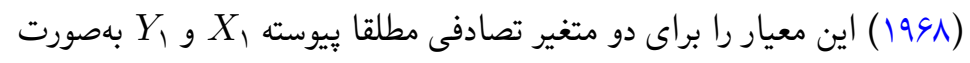

$$
I\left(X_{\backslash}, Y_{\uparrow}\right)=-\int_{x \in S_{X_{1}} \cap S_{Y_{\curlywedge}}} f_{\uparrow}(x) \log g_{\uparrow}(x) d x
$$

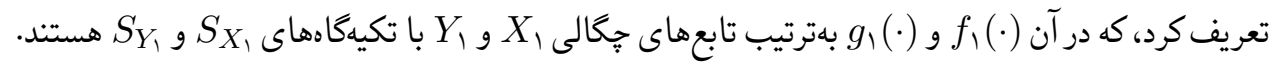

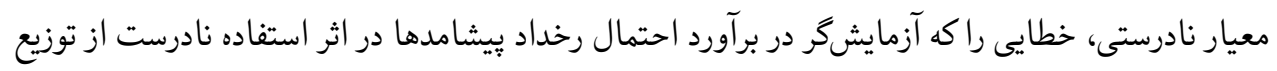
م

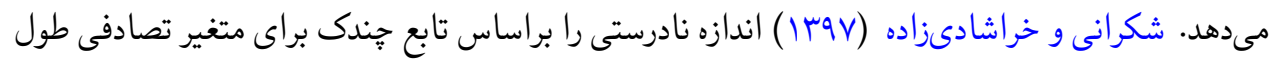

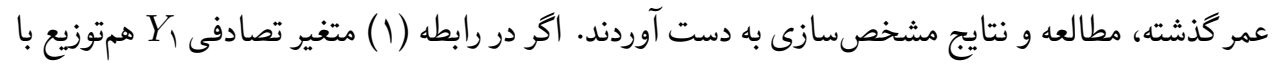
تصاد

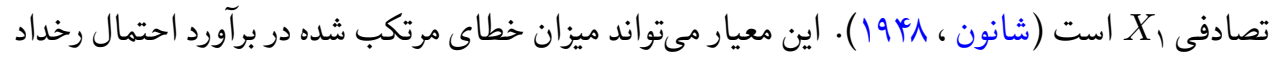

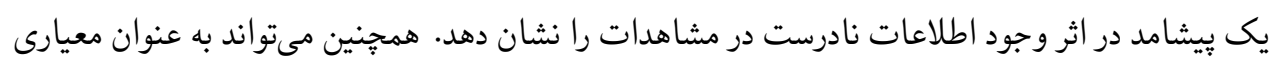

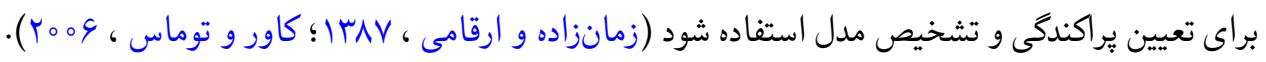
فرض كنيد توأم f و و و تكية كاههاى توأم باشند طورىك نادرستى در حالت دو متغيره بهصورت

$$
\begin{aligned}
& B I(\boldsymbol{X}, \boldsymbol{Y})=-\int_{\ell_{X_{1}}}^{r_{X_{1}}} \int_{\ell_{X_{Y}}}^{r_{X_{Y}}} f(x, y) \log g(x, y) d y d x \\
& \text { است. جاش و كاندو (Y019) نادرستى مانده تجمعى دومتغيره را بهصورت } \\
& \operatorname{BCRI}(\boldsymbol{X}, \boldsymbol{Y})=-\int_{\ell_{X_{1}}}^{r_{X_{1}}} \int_{\ell_{X_{\uparrow}}}^{r_{X_{\uparrow}}} \bar{F}(x, y) \log \bar{G}(x, y) d y d x
\end{aligned}
$$

تعريف كردند، كه در آن حالتى كه Y Y X

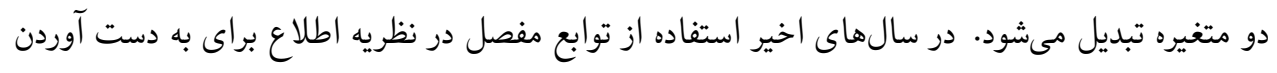




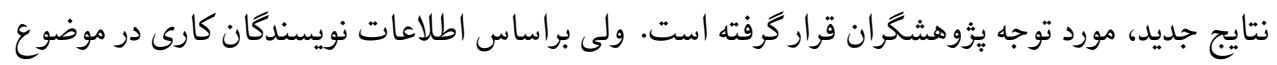

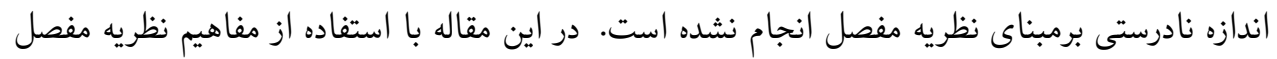

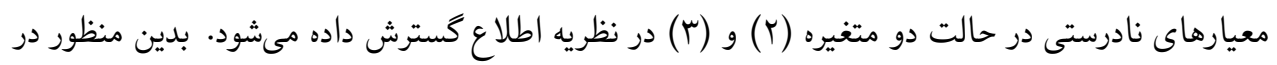

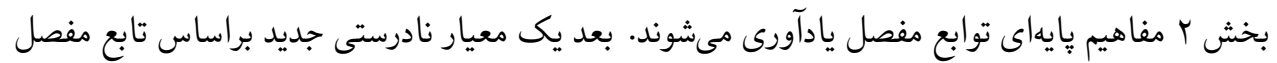

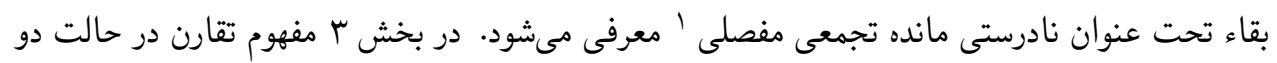

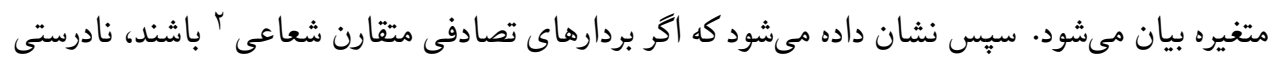

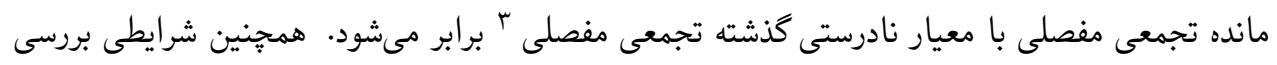

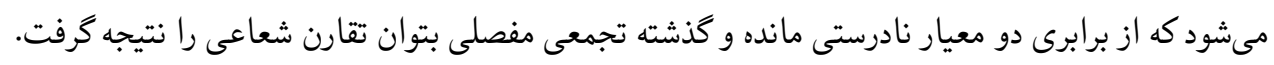

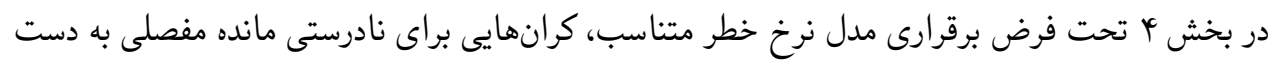

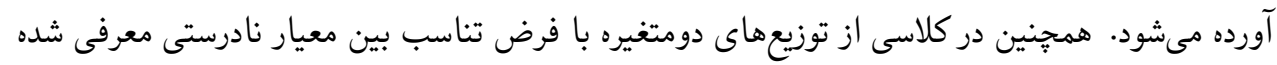

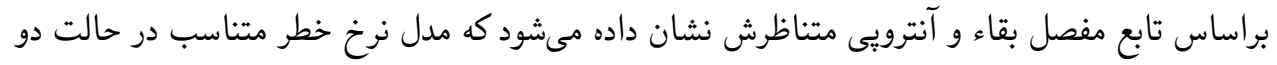

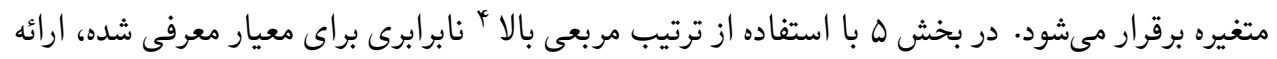

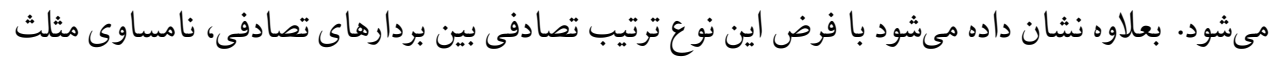

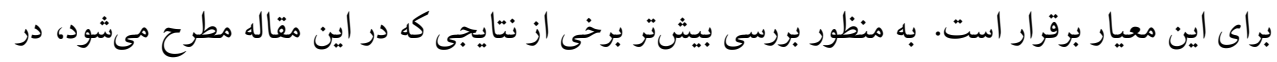

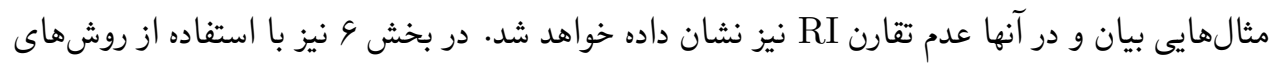

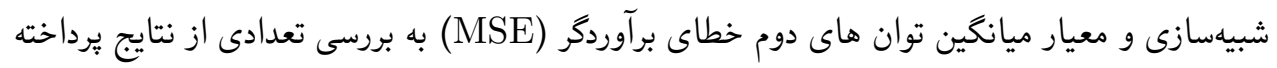

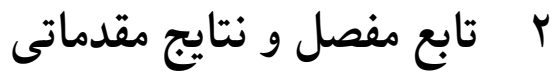

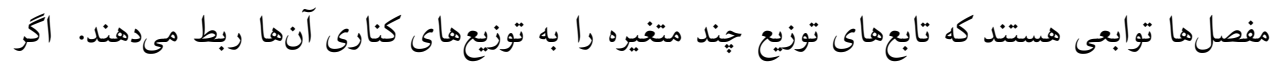

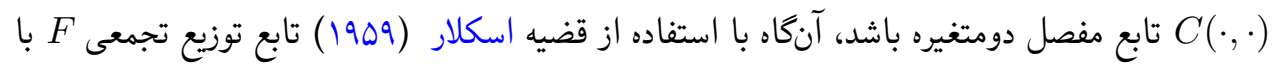

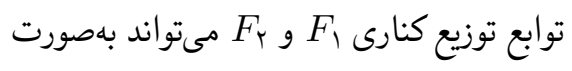

$$
F(x, y)=C\left(F_{\backslash}(x), F_{\Upsilon}(y)\right), \quad x, y \in \overline{\mathbb{R}}=[-\infty,+\infty],
$$

${ }^{1}$ Copula cumulative residual inaccuracy

${ }^{2}$ Radially symmetric

${ }^{3}$ Copula cumulative past inaccuracy

${ }^{4}$ Upper orthant order 
معيار نادبرستى بر مبناى تابع مفصل بقاء

مرتبط شود. تابع مفصل بقاء با نماد $\widehat{~ م ن ~ C ش ا ن ~ د ا د ه ~ م ى ش و د ~ ك ه ~ ب ا ~ ت ا ب ع ~ م ف ص ل ~ ب ه ص و ر ت ~}$

$$
\widehat{C}(u, v)=-1+u+v+C(1-u, 1-v),
$$

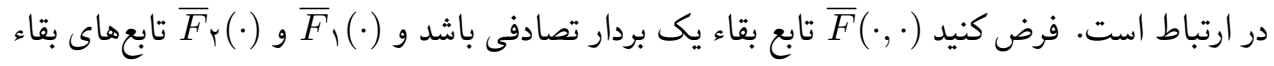

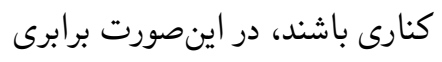

$$
\begin{aligned}
& \bar{F}(x, y)=\widehat{C}\left(\bar{F}_{\uparrow}(x), \bar{F}_{\curlyvee}(y)\right), \quad x, y \in \overline{\mathbb{R}}=[-\infty,+\infty],
\end{aligned}
$$

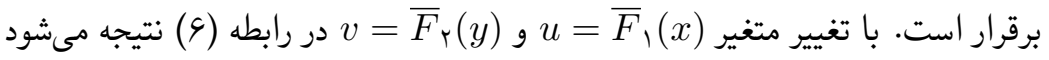

$$
\begin{aligned}
& \widehat{C}(u, v)=\bar{F}\left(\bar{F}_{Y}^{-1}(u), \bar{F}_{r}^{-1}(v)\right) . \\
& \text { براى جزئيات بيشتر درباره نظريه مفصل و كاربردهاى آن به نلسن (Y०Y) مراجعه شود. }
\end{aligned}
$$

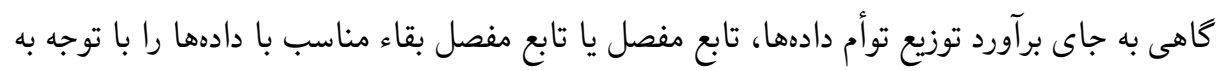

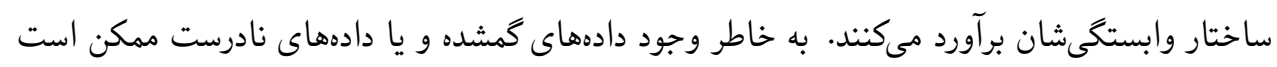

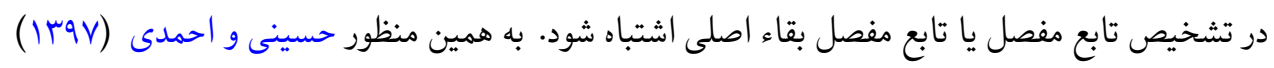

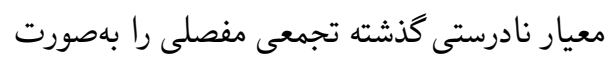
$P I(\boldsymbol{X}, \boldsymbol{Y})=-\int_{0}^{1} \int_{0}^{1} C_{\boldsymbol{X}}(u, v) \log C_{\boldsymbol{Y}}\left(G_{\uparrow}\left(F_{\uparrow}^{-1}(u)\right), G_{\Upsilon}\left(F_{\Upsilon}^{-1}(v)\right)\right) d v d u, \quad(\wedge)$ معرفى كردند. اين معيار وقتى كاربرد دارد كه به جاى استفاده از تابع مفصل درست (·, (·) كاربرد

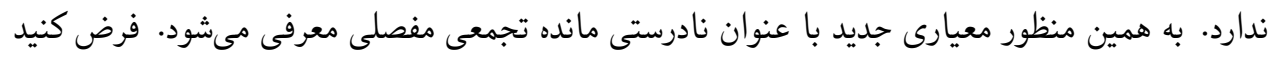
توابع مفصل بقاء

$$
\bar{F}(x, y)=\widehat{C}_{\boldsymbol{X}}\left(\bar{F}_{\uparrow}(x), \bar{F}_{\curlyvee}(y)\right) \quad, \quad \bar{G}(x, y)=\widehat{C}_{\boldsymbol{Y}}\left(\bar{G}_{\uparrow}(x), \bar{G}_{\curlyvee}(y)\right) .
$$


تعريف مىشود. جون

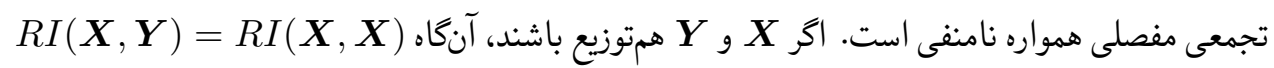

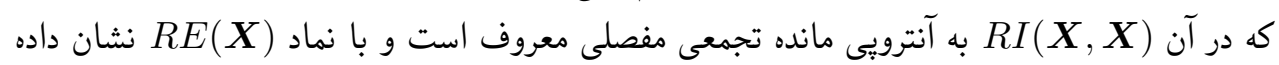

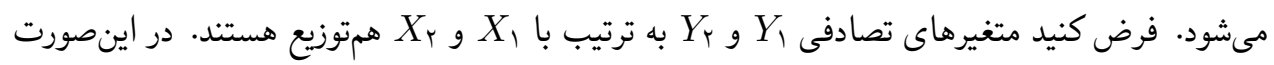
معيار (9) به

$$
R I(\boldsymbol{X}, \boldsymbol{Y})=-\int_{0}^{1} \int_{0}^{1} \widehat{C}_{\boldsymbol{X}}(u, v) \log \widehat{C}_{\boldsymbol{Y}}(u, v) d v d u
$$

تبديل مىشود، كه در بخشهاى بعدى نتايجى براى آن بيان مىشود.

\section{r نتايجى تحت مفروضات تقارن}

تعريف ا (تقارن شعاعى). بردار تصادفى

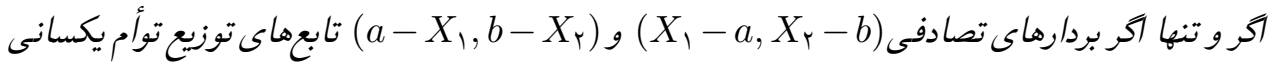
داشته باشند. به عبارتى ديكر، براى هر

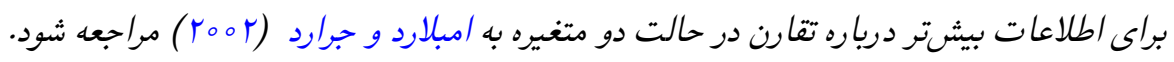
كزاره 1 . فرض كنيد تكيه گاه بردارهاى تصادفى X و

$$
S=(a-c, a+c) \times(b-d, b+d), \quad a, b \in \mathbb{R}, \quad c, d>\circ,
$$

$$
\text { باشد. اكر } \mathbf{X} I(\boldsymbol{X}, \boldsymbol{Y})=P I(\boldsymbol{X}, \boldsymbol{Y}) \text { وتتقارن شعاعى باشند، آنغاه }
$$

برهان: بنابر تقارن شعاعى بردارهاى تصادفى،

$$
F(\ulcorner a-x, \curlyvee b-y)=\bar{F}(x, y), \quad G(\ulcorner a-x, \uparrow b-y)=\bar{G}(x, y), \quad(x, y) \in S
$$




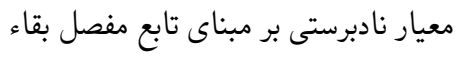

بنابراين از رابطههاى (V) و (9) نتيجه مىشود

$$
\begin{aligned}
R I(\boldsymbol{X}, \boldsymbol{Y}) & =-\int_{a-c}^{a+c} \int_{b-d}^{b+d} f_{\uparrow}(x) f_{\curlyvee}(y) \bar{F}(x, y) \log \bar{G}(x, y) d y d x \\
& =-\int_{a-c}^{a+c} \int_{b-d}^{b+d} f_{\uparrow}(\Upsilon a-z) f_{\curlyvee}(\Upsilon b-w) F(z, w) \log G(z, w) d w d z .
\end{aligned}
$$

از طرفى ديكر، جون بردار تصادفى X متقارن شعاعى است با توجه به (†) و (9) مىتوان برابرى

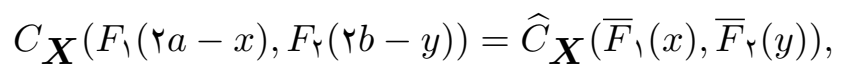

را به دست آورد. براى بردار تصادفى و RI 1 به دست مئيد.

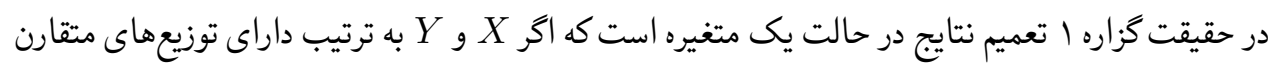

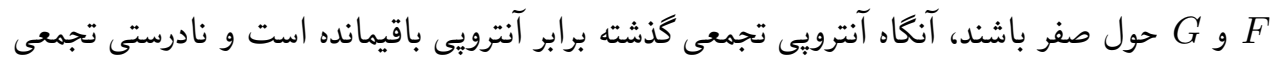

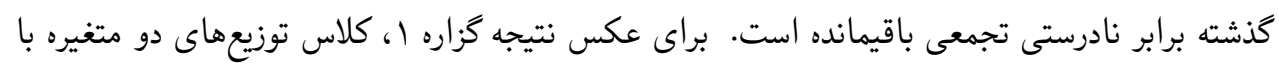
تكيةكاه

$$
C_{\uparrow}=\{H: \bar{H}(a+x, b+y) \leq \iota \geq H(a-x, b-y),\}
$$

را درنظر بخيريد، كه در آن >a,b

كزاره r. فرض كنيد

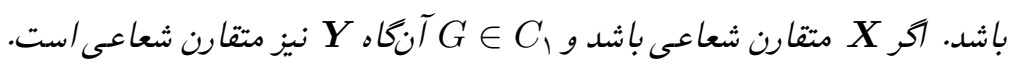

$$
\text { برهان: با استفاده از (9) و (9) داريم }
$$

$$
R I(\boldsymbol{X}, \boldsymbol{Y})=-\int_{a-c}^{a+c} \int_{b-d}^{b+d} f_{\uparrow}(x) f_{\curlyvee}(y) \bar{F}(x, y) \log \bar{G}(x, y) d y d x .
$$




$$
\begin{aligned}
& \text { همجنين با استفاده از (Y) و (^) داريم } \\
& P I(\boldsymbol{X}, \boldsymbol{Y})=-\int_{a-c}^{a+c} \int_{b-d}^{b+d} f_{\backslash}(x) f_{Y}(y) F(x, y) \log G(x, y) d y d x \\
& \text { با توجه به فرض (RI(X, } \\
& \int_{a-c}^{a+c} \int_{b-d}^{b+d} f_{\backslash}(x) f_{\Upsilon}(y) \bar{F}(x, y) \log \bar{G}(x, y) d y d x= \\
& \int_{a-c}^{a+c} \int_{b-d}^{b+d} f_{\backslash}(x) f_{\uparrow}(y) F(x, y) \log G(x, y) d y d x \\
& \text { جون X Xتقارن شعاعى است، داريم } \\
& \int_{-c}^{c} \int_{-d}^{d} f_{\uparrow}(a-z) f_{\uparrow}(b-w) F(a-z, b-w) \log \frac{G(a-z, b-w)}{\bar{G}(a+z, b+w)} d w d z=\circ
\end{aligned}
$$

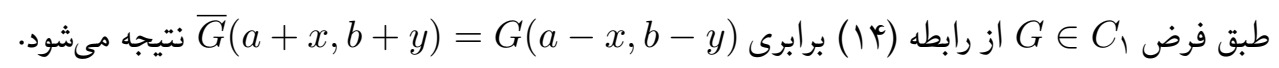

\section{ن ب ايجى تحت فرض مدل نرخ خطرات متناسب}

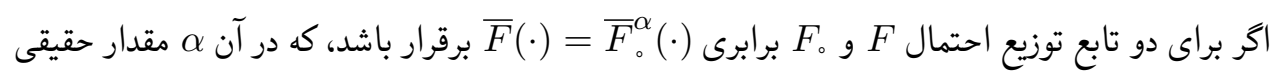

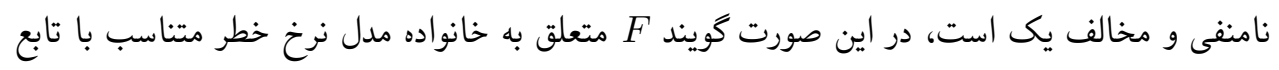

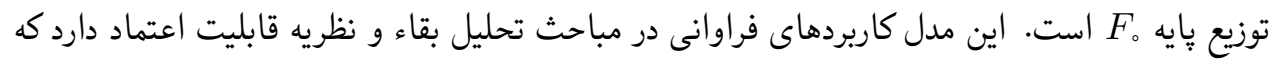

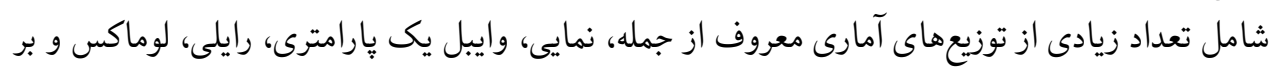

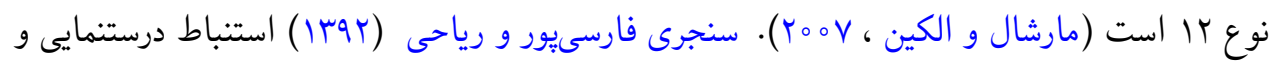

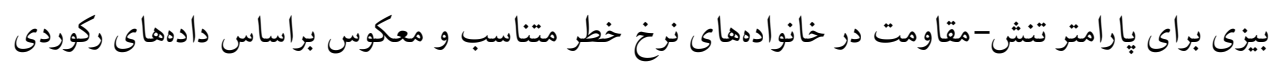

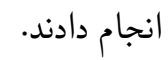

كزاره r. آكر متغيرهاى تصادفى Y و Y Y مستقل از يكديكر باشند و براى هر > > 1 داشته باشيم 
معيار نادبرستى بر مبناى تابع مفصل بقاء

.

$$
\frac{r}{r \varphi}(\alpha+\beta) \leq R I(\boldsymbol{X}, \boldsymbol{Y}) \leq \frac{V}{r \varphi}(\alpha+\beta) \text {. }
$$

$$
\text { برهان: با توجه به رابطه (9) داريم }
$$

$R I(\boldsymbol{X}, \boldsymbol{Y})=-\int_{0}^{1} \int_{0}^{1} \widehat{C}_{\boldsymbol{X}}(u, v) \log \widehat{C}_{\boldsymbol{Y}}\left(\bar{G}_{1}\left(\bar{F}_{Y}^{-1}(u)\right), \bar{G}_{Y}\left(\bar{F}_{Y}^{-1}(v)\right)\right) d v d u$.

با استفاده از نامساوى فرشه-هافدينگگ مىشود

$$
\begin{aligned}
R I(\boldsymbol{X}, \boldsymbol{Y}) \leq & -\int_{0}^{1} \int_{0}^{1} \min \{u, v\} \log \widehat{C}_{\boldsymbol{Y}}\left(\bar{G}_{\uparrow}\left(\bar{F}_{Y}^{-1}(u)\right), \bar{G}_{Y}\left(\bar{F}_{\Gamma}^{-1}(v)\right)\right) d v d u \\
= & -\int_{0}^{1} \int_{0}^{u} v \log \widehat{C}_{\boldsymbol{Y}}\left(\bar{G}_{1}\left(\bar{F}_{Y}^{-1}(u)\right), \bar{G}_{\Upsilon}\left(\bar{F}_{Y}^{-1}(v)\right)\right) d v d u \\
& -\int_{0}^{1} \int_{u}^{1} u \log \widehat{C}_{\boldsymbol{Y}}\left(\bar{G}_{Y}\left(\bar{F}_{Y}^{-1}(u)\right), \bar{G}_{Y}\left(\bar{F}_{Y}^{-1}(v)\right)\right) d v d u . \\
& \\
&
\end{aligned}
$$

$$
\begin{aligned}
& R I(\boldsymbol{X}, \boldsymbol{Y}) \leq-\int_{\ell_{X_{1}}}^{r_{X_{1}}} f_{\uparrow}(x) \log \bar{G}_{\uparrow}(x) \int_{F_{\Upsilon}^{-1}\left(F_{\uparrow}(x)\right)}^{r_{X_{\curlyvee}}} f_{\curlyvee}(y) \bar{F}_{\Upsilon}(y) \log \bar{G}_{\curlyvee}(y) d y d x \\
& -\int_{\ell_{X_{1}}}^{r_{X_{\uparrow}}} f_{\uparrow}(x) \int_{F_{\curlyvee}^{-1}\left(F_{\uparrow}(x)\right)}^{r_{X_{\curlyvee}}} f_{\curlyvee}(y) \bar{F}_{\curlyvee}(y) \log \bar{G}_{\Upsilon}(y) d y d x \\
& -\int_{\ell_{X_{1}}}^{r_{X_{1}}} f_{\uparrow}(x) \bar{F}_{\uparrow}(x) \log \bar{G}_{\uparrow}(x) \int_{\ell_{X_{\curlyvee}}}^{F_{\curlyvee}^{-1}\left(F_{\uparrow}(x)\right)} f_{\curlyvee}(y) d y d x \\
& -\int_{\ell_{X_{1}}}^{r_{X_{1}}} f_{\uparrow}(x) \bar{F}_{\uparrow}(x) \int_{\ell_{X_{Y}}}^{F_{\Upsilon}^{-1}\left(F_{\uparrow}(x)\right)} f_{\Upsilon}(y) \log \bar{G}_{Y}(y) d y d x .
\end{aligned}
$$

${ }^{1}$ Frechet-Hoeffding inequality 
rqv حسينى، س. ت. و احمدى، ج.

با قرار دادن

$$
R I(\boldsymbol{X}, \boldsymbol{Y}) \leq \frac{\vee}{\mu_{\varphi}}(\alpha+\beta)
$$

به دست مى آيد. جون نامساوى $\max \{u+v-1, \circ\} \leq C(u, v)$ براى هر تابع مفصل (·, (·) جمله مفصل بقاء (·, $R I(\boldsymbol{X}, \boldsymbol{Y}) \geq-\int_{0}^{1} \int_{0}^{1} \max \{u+v-1, \circ\} \log \widehat{C}_{\boldsymbol{Y}}\left(\bar{G}_{1}\left(\bar{F}_{1}^{-1}(u)\right), \bar{G}_{Y}\left(\bar{F}_{Y}^{-1}(v)\right)\right) d v d u$ $=-\int_{0}^{1} \int_{1-u}^{1}(u+v-1) \log \widehat{C}_{\boldsymbol{Y}}\left(\bar{G}_{1}\left(\bar{F}_{1}^{-1}(u)\right), \bar{G}_{Y}\left(\bar{F}_{Y}^{-1}(v)\right)\right) d v d u$.

به طور مشابه با (10) نابرابرى (10)

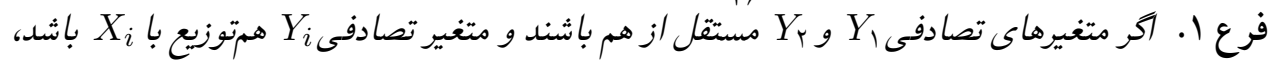

$$
\frac{r}{r \varphi} \leq R I(\boldsymbol{X}, \boldsymbol{Y}) \leq \frac{1 \psi}{r \varphi}
$$

كزاره ץ. أخر

$$
\text { متناسب صدق كنند، آنكاه. }
$$$$
\text { برهان: از (9) و (9) داريم }
$$

$$
R I(\boldsymbol{X}, \boldsymbol{Y})=-\int_{\ell_{X_{1}}}^{r_{X_{1}}} \int_{\ell_{X_{\Upsilon}}}^{r_{X_{\curlyvee}}} f_{\uparrow}(x) f_{\Upsilon}(y) \bar{F}(x, y) \log \bar{G}(x, y) d y d x
$$

$$
\text { با قرار دادن (x, }
$$

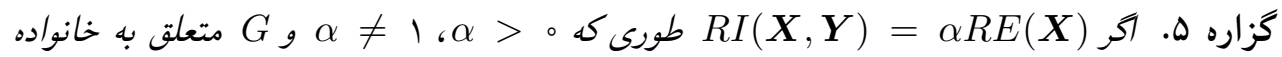
و $\boldsymbol{X}$ و مدل نرخ خطرات متناسب بهصورت $\bar{G}(x, y)=\bar{F}^{\alpha}(x, y)$ برهان: بنا به فرض (X) 
معيار نادبرستى بر مبناى تابع مفصل بقاء ...

rqu

$$
\begin{aligned}
& R E(\boldsymbol{X})=R I(\boldsymbol{X}, \boldsymbol{X}) \\
& \int_{\ell_{X_{1}}}^{r_{X_{\curlywedge}}} \int_{\ell_{X_{\curlyvee}}}^{r_{X_{\uparrow}}} \bar{F}(x, y) f_{\uparrow}(x) f_{\curlyvee}(y) \log \frac{\bar{G}(x, y)}{\bar{F}^{\alpha}(x, y)} d y d x=\circ .
\end{aligned}
$$

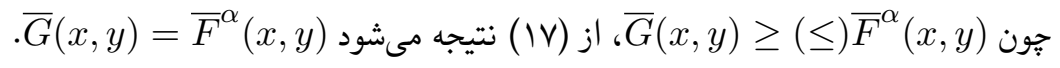

\section{RI ن متايجى براساس ترتيب مربعى بالا براى}

تعريف r (ترتيب مربعى بالا). بردار تصادفى X را در ترتيب مربعى بالا، كوجکتر يا مساوى با بردار

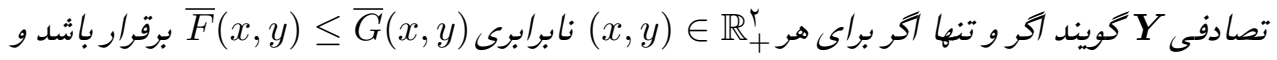
با نماد

براى كسب اطلاعات درباره ترتيبهاى تصادفى به شيكد و شانتىكومار (Y०V) مراجعه شود.

$$
\text { كزاره צ. آحر }
$$

برهان: با توجه به فرض

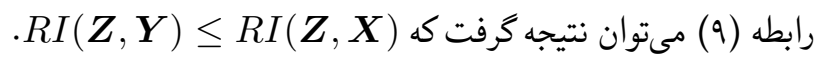

كزاره V. آكر

$$
R I(\boldsymbol{X}, \boldsymbol{Z}) \leq R I(\boldsymbol{Y}, \boldsymbol{Z})
$$

برهان: اثبات مشابه برهان گزاره 9 است. كزاره ^. براى بردارهاى تصادفى X X با الف-آى ب-كر

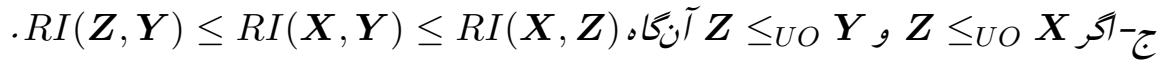


499

حسينى، س. ت. و احمدى، ج.

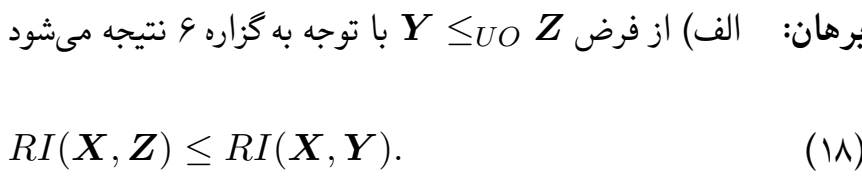

از طرفى ديكر، از فرضهاى توجه به كزاره V مىتوان نتيجه گرفت

$R I(\boldsymbol{X}, \boldsymbol{Y}) \leq R I(\boldsymbol{Z}, \boldsymbol{Y})$

$$
\begin{aligned}
& \text { از نامساوىهاى (1) و (19) داريم (19) } \\
& \text { برهان قسمتهاى ب و بّ نيز مانند قسمت الف بيان مىشود. }
\end{aligned}
$$

كزاره 9.

الف- $R I(\boldsymbol{Z}, \boldsymbol{Y}) \leq R I(\boldsymbol{Z}, \boldsymbol{X})+R I(\boldsymbol{X}, \boldsymbol{Y})$

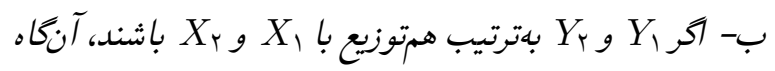

$$
R I(\boldsymbol{X}, \boldsymbol{Z}) \leq R I(\boldsymbol{X}, \boldsymbol{Y})+R I(\boldsymbol{Y}, \boldsymbol{Z})
$$

برهان: اثبات الف با استفاده از كزاره و و نامنفى بودن RI

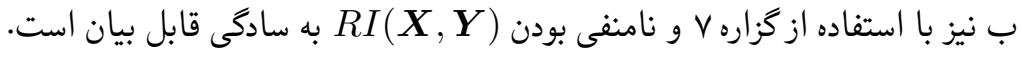

مثال ا. فرض كنيد

$$
\widehat{C}_{\boldsymbol{X}}(u, v)=u v(1+\theta(1-u)(1-v)) \quad, \quad \widehat{C}_{\boldsymbol{Y}}(u, v)=\frac{u v}{1-\lambda(1-u)(1-v)}
$$

باشند، كه درآن آ

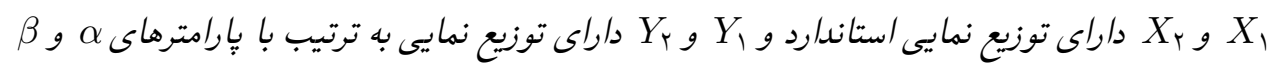


معيار نادبرستى بر مبناى تابع مفصل بقاء

باشند. با استفاده /ز رابطه (9) داريم

$R I(\boldsymbol{X}, \boldsymbol{Y})=-\int_{0}^{1} \int_{0}^{1} u v(1+\theta(1-u)(1-v)) \log \frac{u^{\alpha} v^{\beta}}{1-\lambda\left(1-u^{\alpha}\right)\left(1-v^{\beta}\right)} d v d u$,

كه به م، ر، 0 و $\lambda$ و وابسته /ست. رفتار معيار بالا نسبت به ٍإرامترها بهصورت تحليلى به سادكى قابل بررسى نيست. همان طوركه در شكل ا ملاحظه مئشود (RI

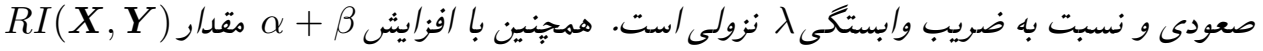
افزايش مىيابد. شكل r رفتار (RI

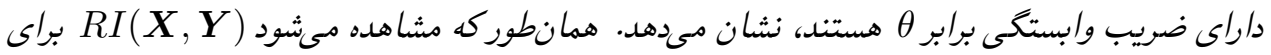

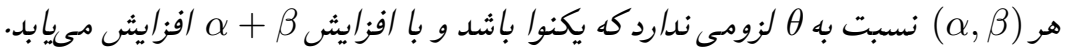

$\alpha=.5, \beta=.5$

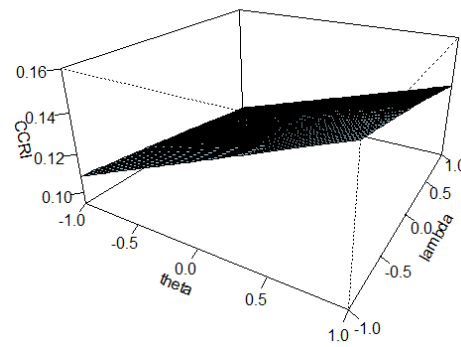

$\alpha=2, \beta=.5$

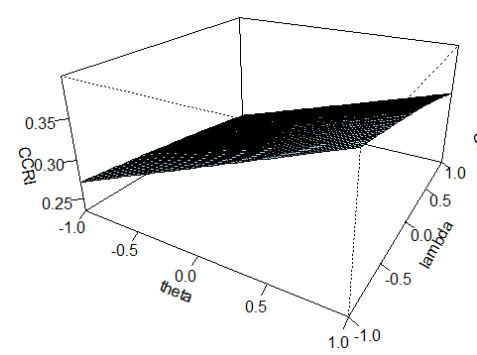

شكل ا. نمودار $R I(\boldsymbol{X}, \boldsymbol{Y}$ برحسب ضرايب وابستگى $\theta$ و $\lambda$

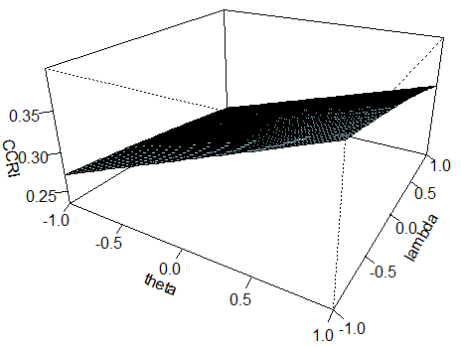

$\alpha=2, \beta=2$

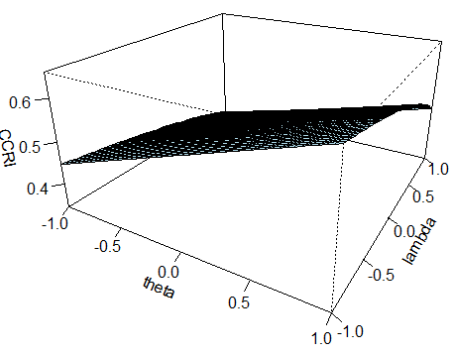

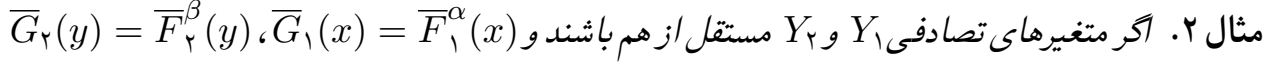


$\varphi \circ 1$

حسينى، س. ت. و احمدى، ج.

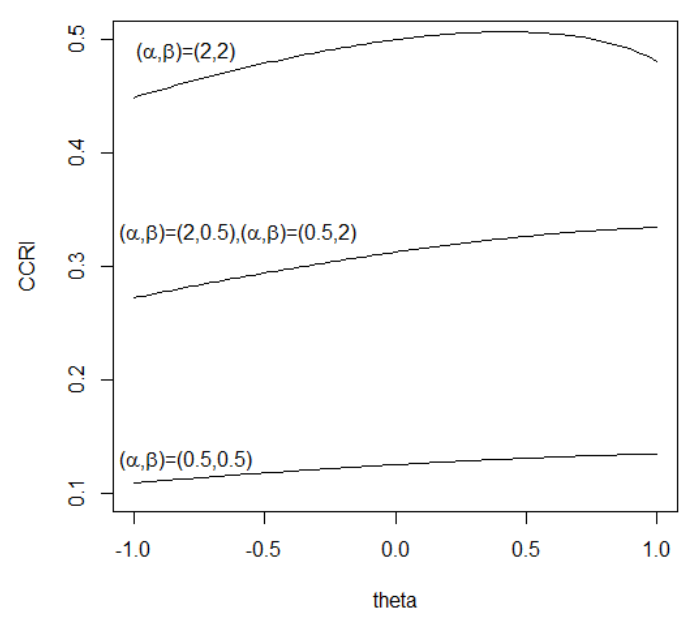

$$
\text { شكل r. نمودار (RI }
$$

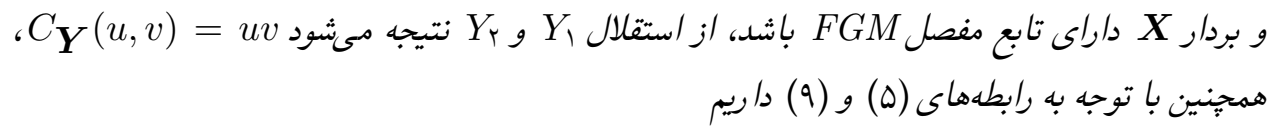

$$
\begin{aligned}
R I(\boldsymbol{X}, \boldsymbol{Y}) & =-\int_{0}^{1} \int_{0}^{1}\{-1+u+v+(1-u)(1-v)(1+\theta u v)\} \log u^{\alpha} v^{\beta} d u d v \\
& =\left(\frac{1}{\wedge}+\frac{\Delta \theta}{r_{1 \varphi}}\right)(\alpha+\beta) .
\end{aligned}
$$

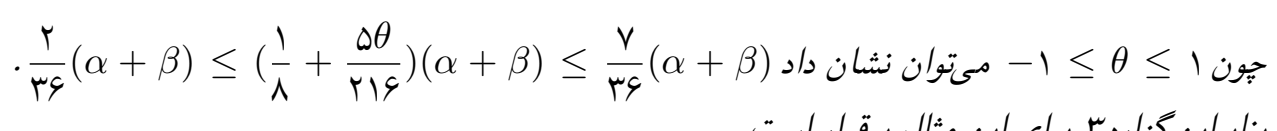
بنابراين گزارها براى اين مثال برقرار است.

مثال ب. فرض كنيد در مثال ب توزيعهاى حاشيهاى نيز متقارن باشند، در اينصورت /ز (ه) و (م) دا ريم

$$
\begin{aligned}
P I(\boldsymbol{X}, \boldsymbol{Y}) & =-\int_{0}^{1} \int_{0}^{1}\{u v(1+\theta(1-u)(1-v))\} \log u^{\alpha} v^{\beta} d u d v \\
& =\left(\frac{1}{\Lambda}+\frac{\Delta \theta}{r_{1 \zeta}}\right)(\alpha+\beta) .
\end{aligned}
$$

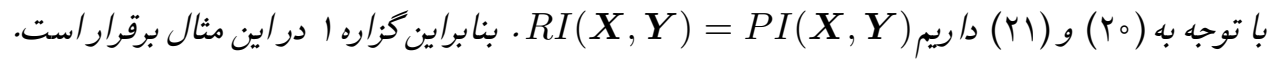


معيار نادبرستى بر مبناى تابع مفصل بقاء

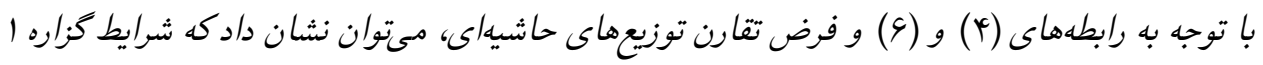
براى بردارهاى تصادفى و بv

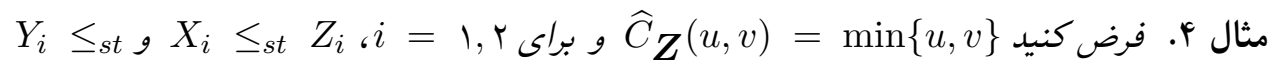

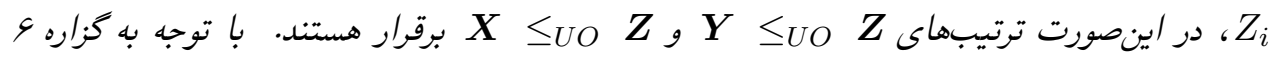
نتيجه مئود (R) $R I(\boldsymbol{Y}, \boldsymbol{Z}) \leq R I(\boldsymbol{Y}, \boldsymbol{X})+g R I(\boldsymbol{X}, \boldsymbol{Z}) \leq R I(\boldsymbol{X}, \boldsymbol{Y})+R I(\boldsymbol{Y}, \boldsymbol{Z})$

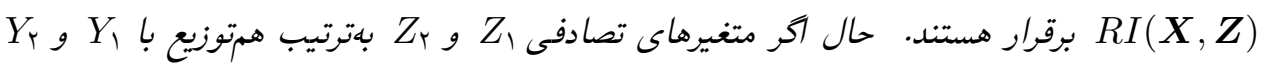
باشند، براساس كزاره V داريم (Z)

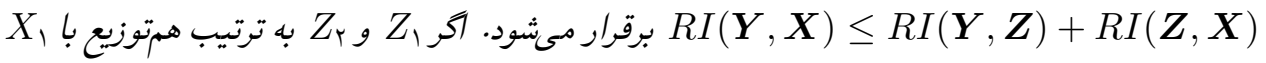
و و بهورت $R I(\boldsymbol{X}, \boldsymbol{Y}) \leq R I(\boldsymbol{X}, \boldsymbol{Z})+R I(\boldsymbol{Z}, \boldsymbol{Y}$ برقرار است.

\section{9}

فرض كنيد حالت برآوردكر تجربى توزيع توأم بهصورت

$$
F_{n}(x, y)=\frac{1}{n} \sum_{i=1}^{n} I\left(X_{i, 1} \leq x, X_{i, r} \leq y\right)
$$

است، كه در آن I تابع نشانكر است. به طور مشابه برآوردكر تجربى براى (x,y) بهصورت

$$
\bar{F}_{n}(x, y)=\frac{1}{n} \sum_{i=1}^{n} I\left(X_{i, 1}>x, X_{i, r}>y\right) .
$$

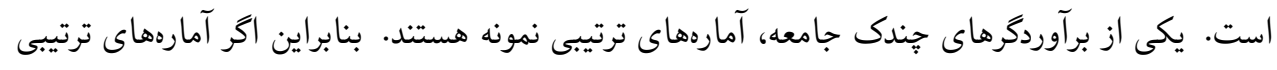

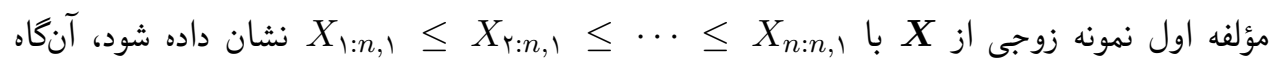


ror

حسينى، س. ت. و احمدى، ج.

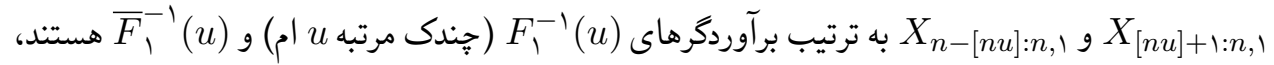

$$
\widehat{F}_{1}^{-1}(u)=X_{[n u]+1: n, 1}, \quad \widehat{\bar{F}}_{1}^{-1}(u)=X_{n-[n u]: n, 1} .
$$

در معيار نادرستى (^) و (9) فرض بر اين است كه تابع مفصل و توزيعهاى حاشيهاى بردار Y

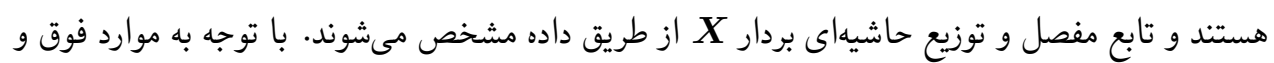

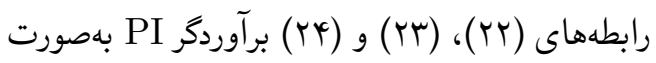

$$
\begin{aligned}
\widehat{P I}(\boldsymbol{X}, \boldsymbol{Y}) & =-\int_{0}^{1} \int_{0}^{1} F_{n}\left(\widehat{F}_{\uparrow}^{-1}(u), \widehat{F}_{ץ}^{-1}(v)\right) \log C_{\boldsymbol{Y}}\left(G_{\uparrow}\left(\widehat{F}_{\uparrow}^{-1}(u)\right), G_{Y}\left(\widehat{F}_{Y}^{-1}(v)\right)\right) d v d u, \\
& =-\int_{0}^{1} \int_{0}^{1} \frac{1}{n} \sum_{i=1}^{n} I\left(X_{i, 1} \leq X_{[n u]+1: n, 1}, X_{i, r} \leq X_{[n v]+1: n, \uparrow}\right) \\
& \times \log C_{\boldsymbol{Y}}\left(G_{1}\left(X_{[n u]+1: n, 1}\right), G_{Y}\left(X_{[n v]+1: n, r}\right)\right) d v d u .
\end{aligned}
$$

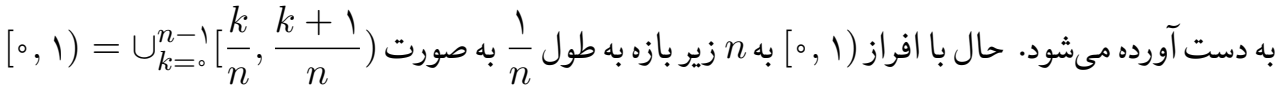
مىتوان نوشت:

$$
\begin{aligned}
\widehat{P I}(\boldsymbol{X}, \boldsymbol{Y}) & =-\sum_{k=0}^{n-1} \sum_{j=0}^{n-1} \int_{\frac{k}{n}}^{\frac{k+1}{n}} \int_{\frac{j}{n}}^{\frac{j+1}{n} \frac{1}{n}} \sum_{i=1}^{n} I\left(X_{i, 1} \leq X_{k+1: n, 1}, X_{i, r} \leq X_{j+1: n, r}\right) \\
& \times \log C_{\boldsymbol{Y}}\left(G_{1}\left(X_{k+1: n, 1}\right), G_{\uparrow}\left(X_{j+1: n, r}\right)\right) d v d u \\
& =-\frac{1}{n^{r}} \sum_{k=0}^{n-1} \sum_{j=0}^{n-1} \sum_{i=1}^{n} I\left(X_{i, 1} \leq X_{k+1: n, 1}, X_{i, r} \leq X_{j+1: n, r}\right) \\
& \times \log C_{\boldsymbol{Y}}\left(G_{1}\left(X_{k+1: n, 1}\right), G_{\Upsilon}\left(X_{j+1: n, r}\right)\right) .
\end{aligned}
$$

$$
\begin{aligned}
\widehat{R I}(\boldsymbol{X}, \boldsymbol{Y}) & =-\frac{1}{n^{\Gamma}} \sum_{k=0}^{n-1} \sum_{j=0}^{n-1} \sum_{i=1}^{n} I\left(X_{i, 1}>X_{n-k: n, 1}, X_{i, r}>X_{n-j: n, r}\right) \\
& \times \log \widehat{C}_{\boldsymbol{Y}}\left(\bar{G}_{1}\left(X_{n-k: n, 1}\right), \bar{G}_{Y}\left(X_{n-j: n, r}\right)\right)
\end{aligned}
$$


كه در آنها n از بردار تصادفى

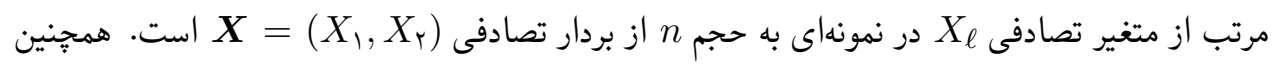

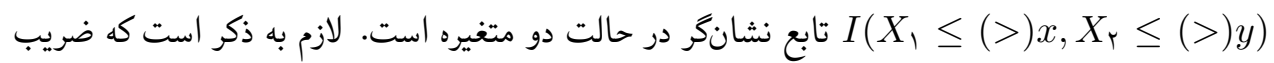
وابستكى در تابع هاى مفصل و مفصل بقاء مربوط به بردار تصادفى Y

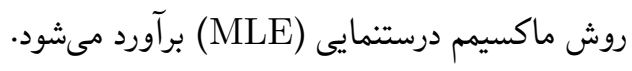
الف- نتيجه حاصل از گزاره ا: فرض كنيد و

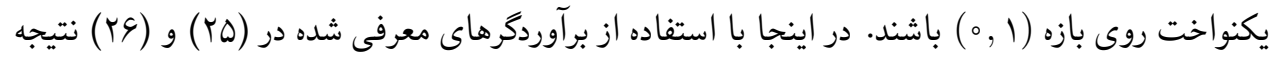

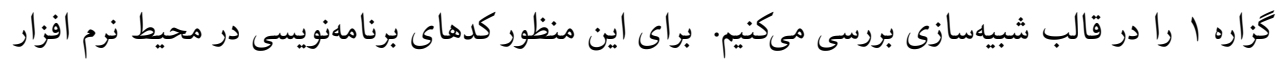

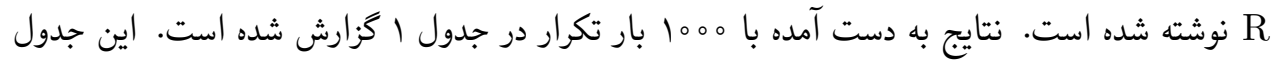
مقادير MSE را براى برآوردگ (

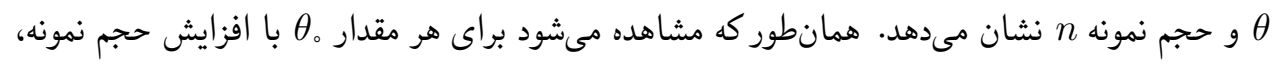

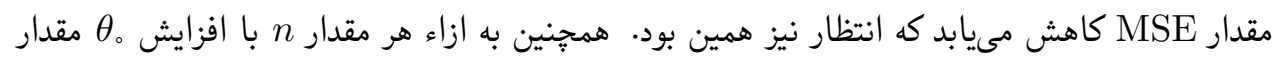

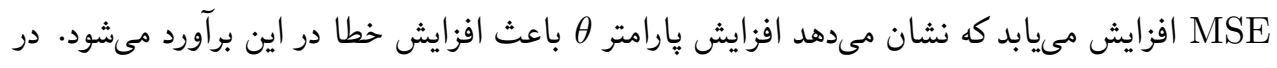

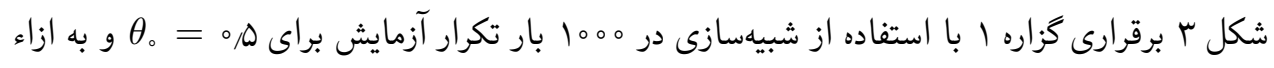

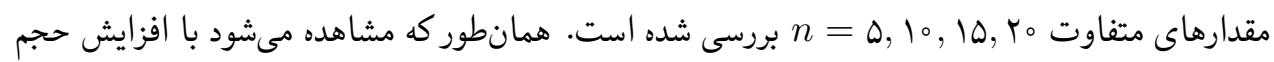
نمونه مقدارهاى شبيهسازى شده |

\begin{tabular}{|c|c|c|c|c|c|}
\hline$M S E$ & $\theta_{0}$ & $n$ & $M S E$ & $\theta_{0}$ & $n$ \\
\hline $0,09 \Delta T Y$ & 0,1 & 0 & $0,09 \vee \wedge 9$ & $\%$ & 0 \\
\hline $0,0 Y 4 \mid 0$ & 0,1 & 10 & 0,04990 & $0 / 0$ & 10 \\
\hline r. & 01 & 10 & 0,01490 & $0 / 0$ & 10 \\
\hline $0,0 \circ Y Y_{0}$ & 0,1 & ro & 0,01079 & 0,0 & ro \\
\hline 0,09114 & $-0,1$ & 0 & $00 \wedge r 9 \Delta$ & $-0, \Delta$ & $\Delta$ \\
\hline OPTYYF & $-0,1$ & 10 & $0,0 Y 0 / F$ & $-0 / \Delta$ & 10 \\
\hline $0,0110 r$ & $-0,1$ & 10 & 0,00991 & $-0, \Delta$ & 10 \\
\hline $0,00 V / \Delta$ & $-0,1$ & ro & 0,00901 & $-\circ \Delta$ & ro \\
\hline . & 。 & 0 & 0,01194 & 。 & 10 \\
\hline O,0YMYY & 。 & 10 & $0,00 V K Y$ & 。 & $r_{0}$ \\
\hline
\end{tabular}

ب- نتيجه حاصل از گزاره با: فرض كنيد 
$\varphi \circ 0$

حسينى، س. ت. و احمدى، ج·
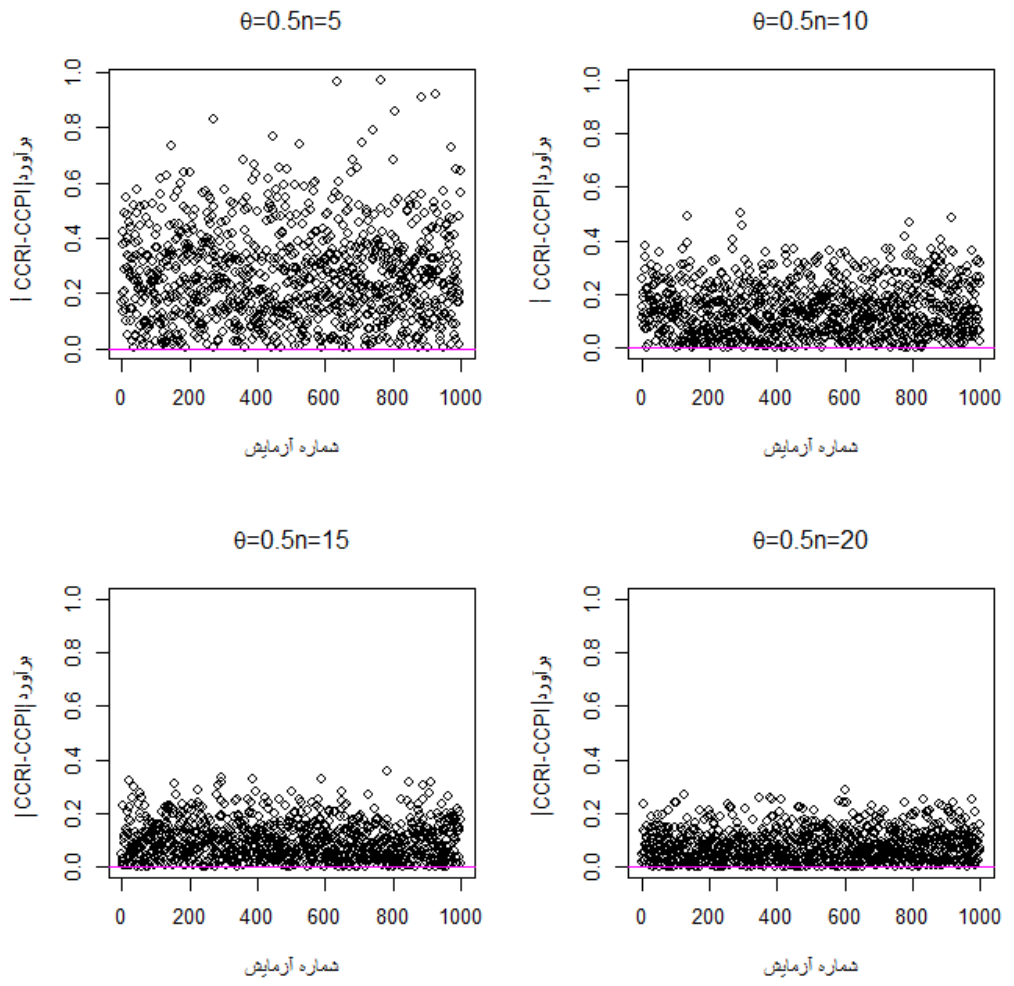

شكل r. مقادير شبيهازى شده |

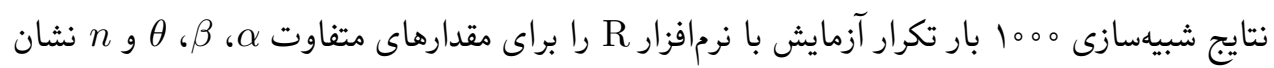

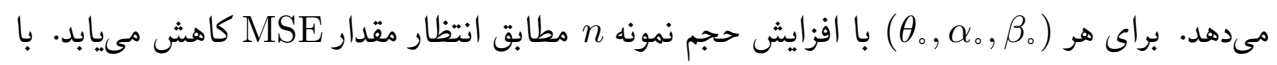

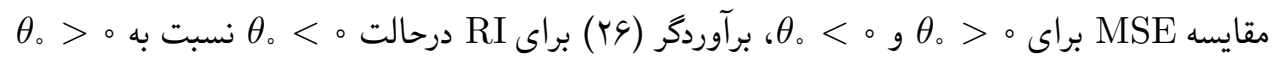

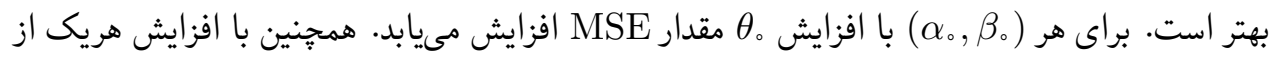

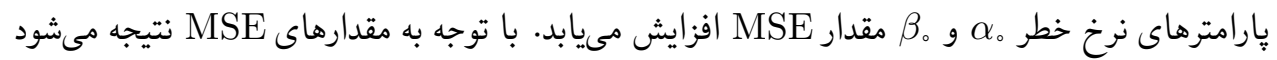

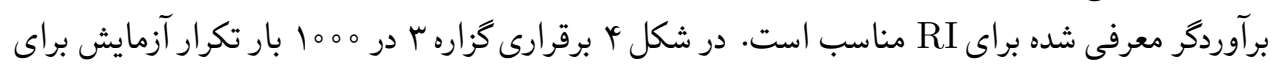

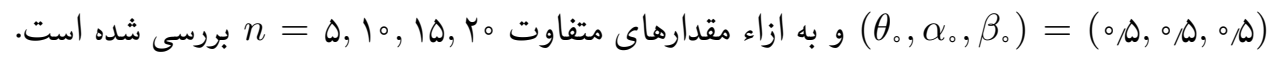
درصد تعداد نقاط قرار گرفته در بازه

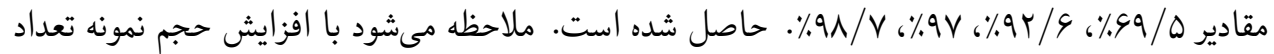
بيشترى از مقادير شبيهسازى شده 


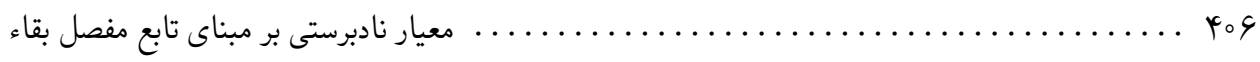

\begin{tabular}{|c|c|c|c|c|c|c|c|c|c|c|c|c|}
\hline$M S E$ & $\beta$ 。 & $\alpha_{\circ}$ & $\theta_{0}$ & $M S E$ & $\beta$ 。 & $\alpha_{\circ}$ & $\theta_{0}$ & $M S E$ & $\beta_{\circ}$ & $\alpha_{\circ}$ & $\theta$ 。 & $n$ \\
\hline $0,0 \circ$ TUY & $\circ \Delta$ & $\%$ & $-0 \pi$ & 0,00419 & $0 \Delta$ & $\circ$ & $0 / 1$ & 0,00494 & $0 / 0$ & $0 /$ & .0 & $\Delta$ \\
\hline $0,001 \mathrm{VT}$ & . & $\% / \Delta$ & $-0 \pi$ & 0,00190 & - ه & $0, \Delta$ & 01 & 0,00194 & $\%$ & ه &.$\Delta$ & 10 \\
\hline 0,00094 & $\Delta$ & $0 / 0$ & $-0 \pi$ & 0,00111 & 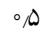 & $0, \Delta$ & 01 & $0,001 Y q$ & $\% 0$ & 0 & $0 \Delta$ & 10 \\
\hline גוTH & r & $0 / \Delta$ & $-0, \pi$ & OOYGVG & r & $0, \Delta$ & $0 / 1$ & $0 \% r \circ 9 \mathrm{~V}$ & $r$ & $\circ \Delta$ &.$\Delta$ & 0 \\
\hline $0,0 \| \Delta Q$ & r & $\circ / \Delta$ & $-0, \pi$ & $0,0 \mid r V V$ & r & $0, \Delta$ & 01 & $0,0 \mid \psi \Delta 0$ & r & . &.$\Delta$ & 10 \\
\hline $0,00 V 9 Y$ & r & $0 / 0$ & $-0 \pi$ & O,OANGD & r & $\circ \Delta$ & 01 & $0,009 \mathrm{VT}$ & r & . D & 0 & 10 \\
\hline ०० YOAY & - & r & $-0 \pi$ & $0,0 \uparrow \Lambda \circ G$ & - D & r & $0 / 1$ & $0,0 \mu_{0} 90$ & $0 / 0$ & r & $0 \Delta$ & 0 \\
\hline 0,01194 & 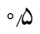 & r & $-0 \pi$ & $0,0 \mid$ THT & ه & r & $0 / 1$ & $0,0 \mid 010$ & $0 / 0$ & r & - $\Delta$ & 10 \\
\hline$\% \circ \circ V \Delta q$ & 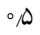 & r & $-0 \pi$ & $0,0 \circ \wedge \Delta 9$ & ه & r & 01 & $0,009 q 4$ & $0 / 0$ & r & - $\Delta$ & 10 \\
\hline $0,0 \Delta \Delta 10$ & r & r & $-0 \pi$ & 0,09419 & r & r & 0,1 & $0,099 V 1$ & r & r & $\circ \Delta$ & $\Delta$ \\
\hline OPYYKA & r & $r$ & $-0, \pi$ & س & r & r & 0,1 & OOMTKY & r & $r$ & .0 & 10 \\
\hline $0,010 H T$ & r & r & $-0 \pi$ & $0,0 \backslash$ NF & r & r & 01 & DOYYYT & r & r &.$\Delta$ & 10 \\
\hline $0,0 \circ \mu \circ \Lambda$ & $\circ \Delta$ & $0 / 0$ & $-0, \Delta$ & O,OOFVG & $\circ$ & $0, \Delta$ & $-0,1$ & $0,0 \circ k 40$ & $0 / 0$ & 0 & 0 & 0 \\
\hline 0,00190 & 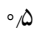 & $0 / 0$ & $-0, \Delta$ & $0,00 \mid \mathrm{VA}$ & 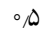 & $0, \Delta$ & $-0,1$ & 0,00190 & $0 / 0$ & 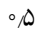 & 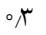 & 10 \\
\hline 0,00091 & ه & $0 / 0$ & $-\circ, \Delta$ & $0,0011 K$ & ه & $0, \Delta$ & $-0,1$ & 0,0 OTS & $0 / 0$ & $\circ \Delta$ & ז & 10 \\
\hline O TrQD & $r$ & $0 / 0$ & $-0, \Delta$ & $0, T \Delta \Delta Q$ & r & $0, \Delta$ & $-0,1$ & \% TVMA & $r$ &.$\Delta$ & ז & 0 \\
\hline $0,010 \mu y$ & r & $0 / 0$ & $-0, \Delta$ & אTHוT & r & 0 & $-0,1$ & $0,0 \mid 4 \circ q$ & r & .0 & 0 & 10 \\
\hline $0,0 \circ V Y \Lambda$ & r & $0 / 0$ & $-0, \Delta$ & $0,0 \circ \wedge k \mid$ & r & . & $-0,1$ & 0,00901 & r & $\circ \Delta$ & 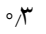 & 10 \\
\hline $0,0 Y M Y O$ & . & $r$ & $-0, \Delta$ & $0,0 Y 990$ & - ه & r & $-0,1$ & \% YA৯০ & $0 / 0$ & $r$ & 0 & 0 \\
\hline $0,010 \wedge V$ & $\circ \Delta$ & r & $-0, \Delta$ & $0,01 K Y 4$ & $\circ$ & r & $-0,1$ & o,olrag & $0 / 0$ & $r$ & 0 & 10 \\
\hline 0,00990 & . & r & $-0, \Delta$ & $0,0 \circ N T$ & . & r & $-0,1$ & $0,0094 \wedge$ & $\%$ & r & $0 \pi$ & 10 \\
\hline $0,0<940$ & r & r & $-0, \Delta$ & 0,09149 & r & r & $-0 / 1$ & $0,09 V A Y$ & r & r & $0 \pi$ & 0 \\
\hline $0,0 Y 191$ & r & r & $-0, \Delta$ & OOTATG & r & r & $-0,1$ & $0,0 \mu|\Lambda|$ & r & r & - & 10 \\
\hline 0,01494 & r & r & $-\circ \Delta$ & 0,01990 & r & r & $-0,1$ & 0,0 YITq & r & r & ז & 10 \\
\hline
\end{tabular}

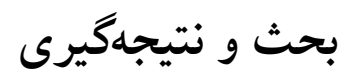

بررسى معيارهاى مختلف براى اندازمخيرى اطلاعات در دادهها يكى از موضوعات مهم و اساسى در زمينههاى

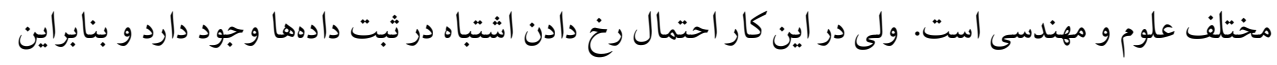

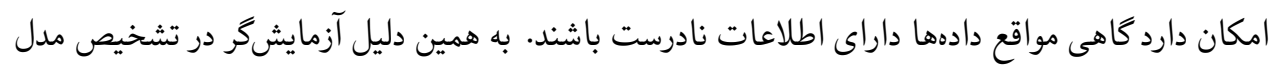

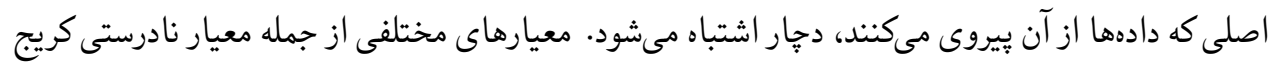

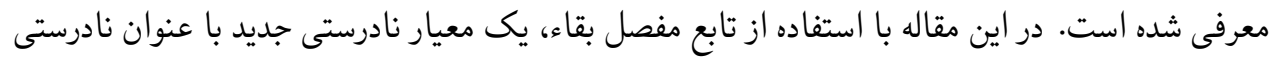

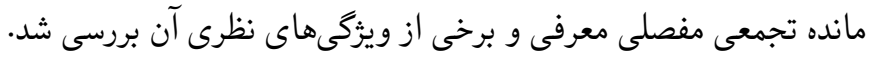

\section{تقدير و تشكر}

نويسندكان از زحمات و ييشنهادات داوران محترم، اعضاى هيئت تحريريه و ويراستار مجله كه باعث بهبود ارائه مقاله شدند، تشكر ميكنتد. 
$r \circ v$
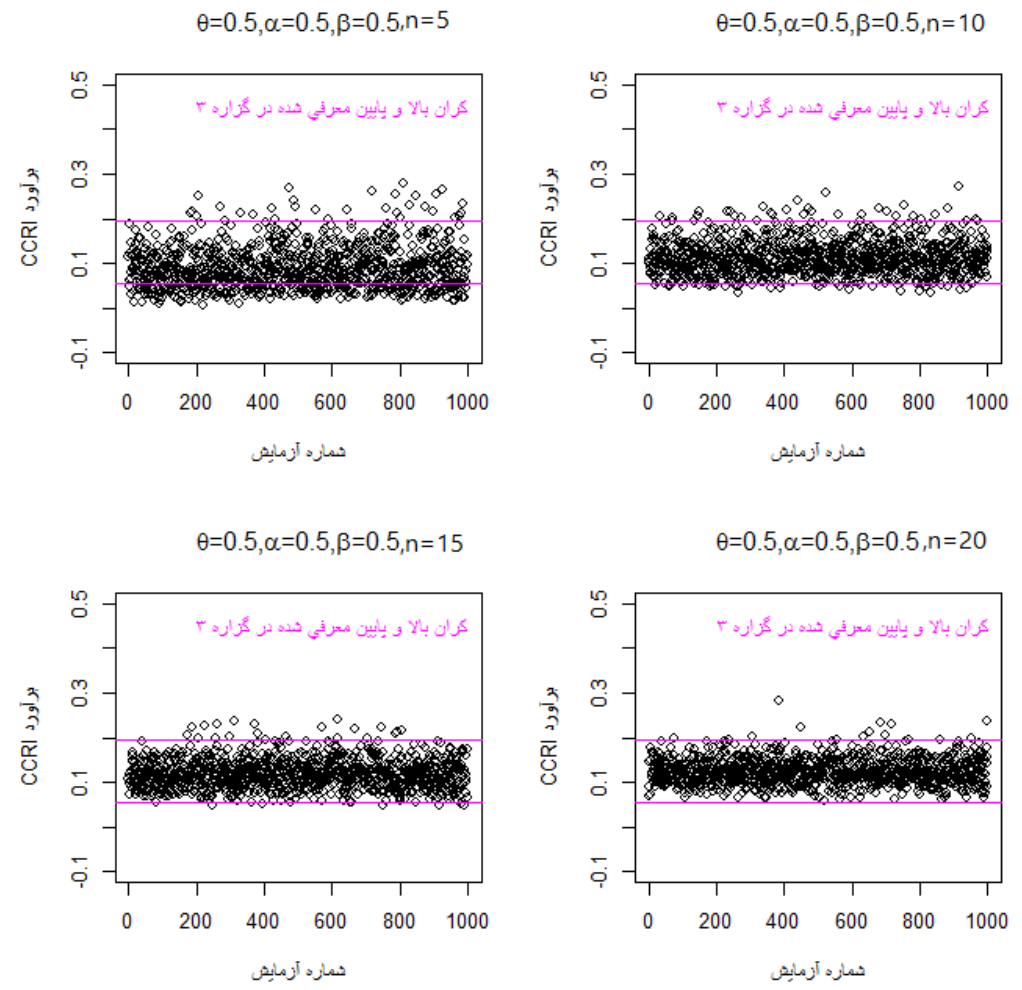

شكل †. مقادير شبيسازى شده

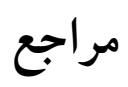

حسينى، ت.، احمدى، ج.، (Y (1))، نتايجى درباره معيار نادرستى در نظريه اطلاع براساس تابع مفصل،

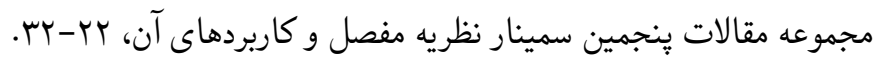

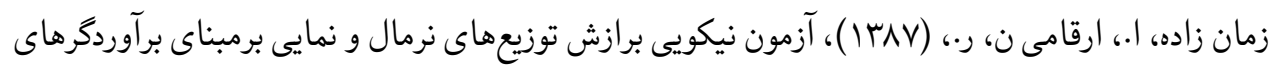

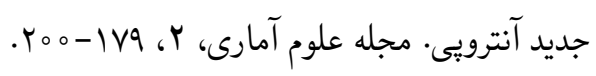

سنجرى فارسىيور، ن، رياحى، ه.، (r (Y I)، استنباط درستنمايى و بيزى مدل تنش نيرو بر اساس داده هاى

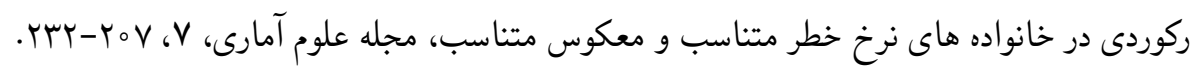

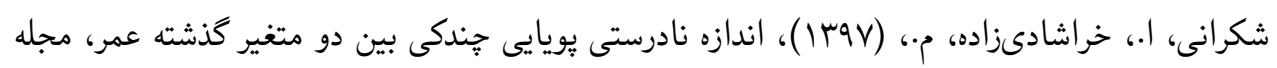


Amblard, C., and Girard, S. (2002) Symmetry and Dependence Properties Within a Semiparametric Family of Bivariate Copulas, Journal of Nonparametric Statistics, 14, 715-727.

Cover, T. M., and Thomas, J. A. (2006), Elements of Information Theory, 2nd edition, Willey-Interscience: NJ.

Ghosh, A. and Kundu, C. (2019), Bivariate Extension of (dynamic) Cumulative Residual and Past Inaccuracy Measures, Statistical Papers, 60, $2225-2252$.

Kerridge, D. F. (1961), Inaccuracy and Inference, Journal of the Royal Statistical Society. Series B (Methodological), 184-194.

Marshall, A. W., and Olkin, I. (2007), Life Distributions, (Vol. 13), Springer, New York.

Nath, P. (1968), Inaccuracy and Coding Theory, Metrika, 13, 123-135.

Nelsen, R. B. (2007), An Introduction to Copulas, Springer, Science and Business Media.

Shaked, M., and Shanthikumar, J. G. (2007), Stochastic Orders, Springer Science and Business Media.

Shannon, C. E. (1948), A Mathematical Theory of Communication, Bell System Technical Journal, 27, 379-423.

Sklar, M. (1959), Fonctions de Repartition an Dimensions et Leurs Marges. Publications de l'Institut de Statistique de l'Université de Paris, 8, 229-231. 
Journal of Statistical Sciences, Spring and Summer, 2020

Vol. 14, No. 2, pp 389-408

DOI: $10.29252 /$ jss.14.2.389

\title{
Inaccuracy Measure Based on Survival Copula
}

Hosseini, S. T. and Ahmadi, J.

Department of Statistics, Faculty of Mathematical Sciences, Ferdowsi University of Mashhad, Mashhad, Iran.

\begin{abstract}
In this paper, using the idea of inaccuracy measure in the information theory, the residual and past inaccuracy measures in the bivariate case are defined based on copula functions. Under the assumption of radial symmetry, the equality of these two criteria is shown, also by the equality between these two criteria, radially symmetrical models are characterized. A useful bound is provided by establishing proportional (inverse) hazard rate models for marginal distributions. Also, the proportional hazard rate model in bivariate mode is characterized by assuming proportionality between the introduced inaccuracy and its corresponding entropy. In addition, orthant orders are used to obtain inequalities. To illustrate the results, some examples and simulations are presented.
\end{abstract}

Keywords: Copula; Entropy; Inaccuracy measure; Orthant orders; Proportional (inverse) hazard rate models; Radially symmetric.

Mathematics Subject Classification (2010): 94A17, 62B10, 60E15. 